\title{
Árvore Binomial Construída via Mapas Randômicos para Análise de Ativos Financeiros
}

\author{
Antonio Airton Carneiro de Freitas* \\ José Roberto Securato**
}

\begin{abstract}
Resumo
Mapas randômicos podem ser construídos a partir do conhecimento a priori sobre o ativo financeiro, por exemplo, utilizando o conhecimento de especialistas ou informação implícita ao mercado. A partir do conhecimento a priori sobre o ativo, identifica-se uma função de distribuição de probabilidades estacionária associada ao seu comportamento dinâmico. O problema inverso também é abordado, ou seja, a partir de uma função de distribuição de probabilidades estacionária empírica ajusta-se um mapa randômico para a construção de uma árvore binomial implícita com a capacidade para incorporar saltos (jumps). Uma aplicação relacionada com o mercado brasileiro de opções é apresentada. Ressalta-se que a qualidade do modelo de incorporar o conhecimento a priori sobre o ativo financeiro pode ser afetada, por exemplo, pela visão enviesada do analista ou por novas mudanças no comportamento dinâmico deste ativo que o modelo não contempla.
\end{abstract}

Palavras-chave: árvores binomiais; opções; mapas randômicos.

Códigos JEL: G13.

\section{Abstract}

Random maps can be constructed from a priori knowledge of the financial assets. It is also addressed the reverse problem, i.e. from a function of an empirical stationary probability density function we set up a random map that naturally leads to an implied binomial tree, allowing the adjustment of models, including the ability to incorporate jumps. An application related to the options market is presented. It is emphasized that the quality of the model to incorporate a priori knowledge of the financial asset may be affected, for example, by the skewed vision of the analyst.

Keywords: binomial trees; options; random maps.

Submetido em Março de 2009. Aceito em Novembro de 2009. O artigo foi avaliado segundo o processo de duplo anonimato além se de ser avaliado pelo editor. Editor responsável: Eduardo Facó Lemgruber.

*FEA - USP, Departamento de Finanças, São Paulo, Brasil. E-mail: airtoncarneiro@utfpr.edu.br

**FEA - USP, Departamento de Finanças, São Paulo, Brasil. E-mail: securato@usp.br 


\section{Introdução}

Na década de 1920, os modelos para ciclos econômicos baseados em equações diferenciais ou a diferenças apresentaram evidências de que a teoria geral do equilíbrio era ambígua (Schenk-Hoppé, 2000). O trabalho pioneiro de Frisch (1933, 1936) corroborou estas evidências com a criação dos conceitos de equilíbrio estático e dinâmico para caracterizar as relações estruturais entre as variáveis que compõe um modelo dinâmico. A Teoria de Newton, com foco determinista, teve inicialmente grande influência sobre as teorias econômicas que a sucederam, culminando com a teoria do valor (Debreu, 1959) e da análise da competitividade (Arrow e Hahn, 1971).

Os conceitos mais importantes da teoria geral do equilíbrio temporário (Grandmont e Hildenbrand, 1974), que se transformou em uma das partes centrais da teoria econômica, inicialmente ainda foram influenciados pela teoria de Newton. Nesse sentido, as pesquisas econômicas eram atemporais, diferentemente do contexto atual que consideram a influência tempo, como por exemplo, os modelos econômicos baseados em processos de Markov. Posteriormente, durante a concepção da teoria geral do equilíbrio, Grandmont $(1977,1982)$ detectou deficiências no enfoque puramente determinista e começou a utilizar também conceitos de processos estocásticos.

Nos primeiros anos das finanças matemáticas moderna, aparentemente sem sofrer influências das teorias econômicas, Cox et alii (1979) propuseram uma árvore binomial para o apreçamento de opções. Esta abordagem consegue lidar com sistemas fortemente estocásticos e com o equilíbrio dinâmico temporário. É bastante flexível já que permite a utilização de vários métodos de ajuste de árvore binomial, transformando-se em uma das favoritas entre os profissionais da área. Este tipo de apreçamento seria uma versão discreta dos modelos de Black e Scholes (1973) e de Merton (1973). Entretanto, este modelo considera que a volatilidade é constante e isto nem sempre é verdadeiro na prática.

Para minimizar este problema da volatilidade permanecer constante surgiram as árvores binomiais implícitas, consistentes com o sorriso da volatilidade, como a apresentada em Rubinstein (1994), e depois em Derman e Kani (1994), Dupire (1994) e Barle e Cakici (1998). Em Chang e Tabak (2002) foram analisados dados do mercado financeiro brasileiro e foi observado que a volatilidade implícita possui conteúdo informacional que não é contemplado pelos modelos baseados em séries de tempo, tais como GARCH $(1,1)$ ou média móvel (MA). Inclusive, a volatilidade implícita agrega informações diferentes e mais efetivas para estimar a volatilidade do ativo subjacente, que são importantes para a construção de cenários.

No contexto de sistemas dinâmicos estocásticos dos quais dispomos apenas de dados empíricos sob a forma de uma função de densidade de probabilidade (fdp) expressa por $f$ e cuja dinâmica subjacente é desconhecida, o problema de determinar uma transformação determinística $\tau$ que aproxima esta dinâmica, sendo que a medida invariante deste sistema é dada por $f$, é referido como o problema inverso de Perron-Frobenius. 
Assim, seja $f$ uma função de densidade de probabilidades constantes em faixas do intervalo $I$. O problema inverso de Perron-Frobenius consiste em determinar uma transformação $\tau: I \rightarrow I$ tal que um sistema unidimensional dinâmico $x_{i+1}=\tau\left(x_{i}\right)$ tem $f$ como a sua única função de densidade invariante. A matriz $M$ que expressa estas transformações é construída de tal maneira que fornece uma simples relação entre $\tau$ e $f$, onde $f$ é qualquer função de densidade constante por cada seção do intervalo unitário. O artigo de Bahsoun et alii (2007) apresenta a metodologia para a modelagem e previsão de dados via mapas randômicos, inclusive de sistemas dinâmicos caóticos (Góra e Boyarsky, 1993, 2003).

A complexidade e o esforço demandado na utilização desta metodologia não são triviais, mas após as rotinas computacionais estiverem testadas, esta metodologia pode tornar-se atrativa. Este esforço para lidar com a complexidade pode ser recompensada com a capacidade de explanação das árvores binomiais geradas, possibilitando análises sobre os possíveis retornos e riscos. A aplicação desta metodologia pode ampliar a visão do analista sobre as possíveis variações que poderão ocorrer no ativo analisado.

O objetivo principal deste artigo é apresentar ao mercado brasileiro a metodologia sugerida no artigo de Bahsoun et alii (2007) que trata da construção de mapas randômicos com dependência da posição para a previsão de ativos financeiros. $\mathrm{O}$ objetivo secundário é ilustrar esta metodologia com uma aplicação no mercado de opções brasileiro.

Este artigo está organizado como segue. Na Seção 2, as bases conceituais necessárias para se alcançar os objetivos são apresentadas. Na Seção 3, descrevese a metodologia adotada na construção das árvores binomiais implícitas com a ilustração de exemplos. Uma aplicação desta metodologia no mercado de opções brasileiro é apresentada na Seção 4. Finalmente, na Seção 5, apresentam-se os comentários finais sobre o método.

\section{Bases Conceituais}

\subsection{Sistemas Dinâmicos Estocásticos}

Os modelos baseados na teoria de sistemas dinâmicos estocásticos são tidos como alternativas atrativas para a modelagem e análise de fenômenos físicos, financeiros e econômicos com componentes estocásticas (Schenk-Hoppé, 2000). Um mapa randômico dependente do tempo é um sistema dinâmico randômico discreto no tempo, consistindo de um conjunto de transformações que, a cada iteração, são selecionadas e aplicadas randomicamente, utilizando probabilidades dependentes da posição. Logo uma árvore binomial ajustada via mapas randômicos pode ser inserida na teoria dos sistemas dinâmicos estocásticos. 
Entretanto, Schenk-Hoppé (2000) acredita que a utilidade desta abordagem na análise do comportamento dinâmico de sistemas de longa duração é mais geral e completa do que aqueles obtidos usando somente equilíbrio de Markov que geralmente é apenas de curta duração. Entretanto, as técnicas de modelagem por meio de sistemas dinâmicos randômicos ainda estão começando na área financeira.

\section{Modelos explícitos de sistemas dinâmicos estocásticos}

A modelagem de variáveis econômicas e financeiras pode depender do entrelaçamento de condições implícitas (comportamento racional dos agentes, por exemplo, o de poupar), logo surgem dificuldades de se encontrar uma representação por meio de modelos explícitos. Entretanto, se a modelagem é guiada pela teoria dos sistemas dinâmicos randômicos desde o início, antes de qualquer abstração, então, acredita-se que o modelo explícito pode ser obtido.

Matematicamente, um modelo de um sistema dinâmico relaciona estados e variáveis em períodos diferentes no tempo e, por definição, requer: (1) definição de um espaço de estados, em particular, os estados iniciais; (2) todas as equações do modelo têm soluções diferentes para diferentes condições iniciais, gerando trajetórias no espaço de estados que nunca se cruzam (permite análise de estabilidade e a condição para que isto ocorra é que as condições iniciais sejam linearmente independentes); e (3) estas soluções exibem uma estrutura no tempo adequada para a dependência entre estados e variáveis. Já a eficácia de um modelo depende principalmente das seguintes condições: modelo satisfatório, ajustado em tempo hábil e a custo barato; medição correta das variáveis exógenas; eficiência dos mercados. O modelo de Black e Scholes (1973) é um exemplo de sucesso.

Uma relação estrutural é dita estática se todas as variáveis se referem ao mesmo instante de tempo; caso contrário a relação é chamada dinâmica. Também foi sugerido em se classificar um sistema como dinâmico se pelo menos ele tiver uma relação estrutural dinâmica. Esta terminologia, um legado de Frisch (1933, 1936), cujo trabalho foi reconhecido com um Prêmio Nobel, é aceito até os dias de hoje e tornou clara porque a teoria geral do equilíbrio é uma relação dinâmica, principalmente depois que foram adicionadas as variáveis que representam os bens e os serviços.

Um modelo econômico é chamado explícito, se ele pode ser descrito por um sistema dinâmico, de acordo com a definição acima; caso contrário, o modelo é chamado implícito. A noção de sistema dinâmico aqui é entendida como um termo genérico para não autônomos (pode ser forçado ou ter um termo perturbador). Neste trabalho, os sistemas dinâmicos randômicos herdam termos, conceitos, métodos e paradigmas da teoria econômica e dos sistemas dinâmicos.

Os modelos de sistemas dinâmicos estocásticos explícitos podem incluir uma perturbação estocástica e ser representados pelas seguintes medidas $(\omega, £, P, \theta)$ que contempla o espaço de probabilidade $P$ junto com um mapeamento $\theta$ mensurável e inversível tal que $\theta P=P$. Chamamos este sistema de ergódigo se $P$ é ergódigo em relação ao mapeamento $\theta$.x 
Considerando a equação a diferenças randômica $x_{t+1}=h\left(\theta^{t} \omega, x_{t}\right)$, onde $\omega$ é uma perturbação, o espaço $X \subset R^{d}$ e o mapeamento $h(\omega, \cdots)=: h(\omega): X \rightarrow$ $X$ é mensurável e o seu inverso também é mensurável, apresenta-se a seguinte definição:

$$
(t, \omega, \chi)=\left\{\begin{array}{l}
h\left(\theta^{t-1} \omega\right) \cdots h(\omega) \chi \quad \text { para } t \geq 1 \\
\chi \text { para } t=0 \\
h\left(\theta^{t} \omega\right)^{-1} \cdots h\left(\theta^{-1} \omega\right)^{-1} \chi \quad \text { para } t \leq-1
\end{array}\right.
$$

onde $\varphi(t, \omega, \chi)$ é o estado do sistema estocástico descrito pelo mapeamento estocástico $h$ no tempo $t$, o qual tem origem em $\chi_{0}=\chi$ sob a perturbação causada por $\omega \theta^{t}=\theta \cdots \theta$. Em particular, $\varphi(1, \omega, \cdot)$ e $h$ é o gerador de $\varphi$. Quando $t \leq-1$ então o mapeamento é inversível, mas, na prática, geralmente só se define $\varphi(t, \omega, \chi)$ para $t \geq 0$. A família de mapeamentos ou estados $\varphi(t, \omega, \chi)$ é chamada de sistema dinâmico estocástico.

Para analisar os modelos explícitos de sistemas dinâmicos estocásticos necessita-se da definição a seguir.

Definition 2.1 Um ponto fixo randômico de um sistema dinâmico estocástico explícito $\varphi(t, \omega, \chi)$ em $X$ é uma variável randômica $\chi^{*}: \Omega \rightarrow X$ tal que, quase sempre,

$$
\chi^{*}(\theta \omega)=\varphi\left(1, \omega, \chi^{*},(\omega)\right):=h\left(\omega, \chi^{*},(\omega)\right)
$$
todo $t$.

Isto implica que $\chi^{*}\left(\theta^{t+1} w\right)=h\left(\theta^{t} \omega, x^{*}\left(\theta^{t} \omega\right)\right)=\varphi\left(t+1, \omega, x^{*}(\omega)\right)$ para

Um ponto fixo randômico é uma solução estacionária de uma equação diferencial ou a diferenças randômicas. Caso a perturbação $\omega$ seja trivial, a definição de ponto fixo coincide com a noção de estado em regime permanente para sistemas dinâmicos determinísticos. Determinar um ponto fixo randômico é equivalente a resolver um número grande de equações acopladas.

\section{3. Árvore Binomial a partir de Mapas Randômicos Dependentes da Posição}

Os mapas randômicos levam naturalmente às árvores binomiais com função de densidade de probabilidade estacionária e podem ser classificados de modelos explícitos de sistemas dinâmicos estocásticos. Este tipo de modelo binomial foi primeiramente explorado na literatura em Bahsoun et alii (2007). Entretanto, este trabalho pioneiro deixou muitos caminhos abertos para serem explorados, inclusive ficou em aberto duas questões importantes: quais são as possíveis aplicações práticas para esta metodologia na área de finanças? Será que o esforço para se lidar com sua complexidade será recompensado pelos resultados obtidos?

\subsection{Mapas randômicos}

O primeiro passo no ajuste de uma árvore binomial via mapas randômicos é criar as leis de formações de up $(u)$ e down $(d)$ ao longo do tempo. Pode-se inclu- 
sive utilizar o conhecimento a priori dos especialistas do mercado financeiro, por exemplo, características observadas nos preços dos ativos como uma correlação negativa entre os retornos do ativo e a volatilidade. No exemplo que segue foram escolhidas leis de formações de $u$ e $d$, que são associadas às funções $\tau u$ e $\tau d$, de tal maneira que a variação dos preços tem uma parcela que é inversamente proporcional ao valor do preço do estado anterior.

A lei de up segue as seguintes regras: caso o ativo esteja muito depreciado, ou seja, seu valor esteja na faixa de $0<x \leq 0,3$, onde $x$ é um valor normalizado do preço do ativo, então ele tem potencial de valorizar $40 \%(1,4)$; já se o ativo estiver na faixa intermediária de $0,3<x \leq 0,7$, ele tem potencial de valorizar $15 \%$ $(1,15)$ mais uma parcela que é inversamente proporcional ao seu valor, ponderada por 1/10; finalmente, quando o ativo está na faixa tem potencial de valorizar $10 \%$ mais uma parcela inversamente proporcional ao seu valor, ponderada por $1 / 5$. A lei de down segue a mesma lógica para sua especificação e é apresentada abaixo. Tanto as leis de up como de down são lineares por faixas dos preços do ativo, resultando em:

$$
u(x)=\left\{\begin{array}{l}
1,4, \quad 0<x \leq 0,3 \\
1,15+\frac{1}{10 x}, \quad 0,3<x \leq 0,7 \\
1,10+\frac{1}{5 x}, \quad 0,7<0 \leq 1
\end{array}\right.
$$

e

$$
d(x)=\left\{\begin{array}{l}
0,9, \quad 0<x \leq 0,3 \\
0,9-\frac{1}{10 x}, \quad 0,3<x \leq 0,7 \\
0,9-\frac{1}{3 x}, \quad 0,7<0 \leq 1
\end{array}\right.
$$

Observa-se que $u(x) \geq 1$ e $d(x) \leq 1$. As probabilidades podem ser atribuídas de acordo com o conhecimento a priori sobre o ativo, por exemplo, neste caso atribuem-se os seguintes valores de probabilidades:

$$
p_{u}(x)= \begin{cases}0,75, & 0<x \leq 0,3 \\ 0,65, & 0,3<x \leq 0,7 \\ 0,60, & 0,7<x \leq 1\end{cases}
$$

e

$$
p_{d}(x)=1-p_{u}(x)
$$

Sendo $p_{u}$ e $p_{d}(x)$ um caso particular da definição genérica dada para um conjunto de probabilidades $\left\{p_{k}(x)\right\}_{k=1}^{K}$, mais ainda, $\sum_{k=1}^{K} p_{k}(x)=1, p_{k}(x) \leq 0$ para qualquer $x \in X$. A partir de $u(x), d(x), p_{u}(x)$ e $p_{d}(x)$, constrói-se o mapa randômico $T=\left\{\tau_{u}(x), \tau_{d}(x) ; p_{u}(x)\right.$ e $\left.p_{d}(x)\right\}$ :

$$
\tau_{u}(x)=\left\{\begin{array}{l}
1,4 x, \quad 0<x \leq 0,3 \\
1,15 x+\frac{1}{10}, \quad 0,3<x \leq 0,7 \\
1,10 x+\frac{1}{5}, \quad 0,7<x \leq 1
\end{array}\right.
$$


e

$$
\tau_{d}(x)=\left\{\begin{array}{l}
0,9 x, \quad 0<x \leq 0,3 \\
0,9 x-\frac{1}{10}, \quad 0,3<x \leq 0,7 \\
0,9 x-\frac{1}{4}, \quad 0,7<x \leq 1
\end{array}\right.
$$

Os valores de $p_{u}(x)$ e $p_{d}(x)$ foram definidos anteriormente. Destaca-se que existe bastante flexibilidade para se determinar os valores de $p_{u}(x)$ e $p_{d}(x)$ como também as leis de up e down, possibilitando bastante liberdade para inserir as peculiaridades de cada ativo.

Observamos que a construção de mapas randômicos deve obedecer às definições e aos teoremas apresentados em Góra e Boyarsky (2003) e Bahsoun et alii (2007). Somente três teoremas serão apresentados neste artigo para uma compreensão melhor e mais rápida de como podemos utilizar esta metodologia.

Theorem 3.1 um mapa randômico $T$ como apresentado anteriormente e $\tau_{k}^{\prime}(x)$ a derivada de $\tau_{k}(x)$. Caso $\sum_{k=1}^{K} \frac{p_{k}(x)}{\left|\tau_{K}^{\prime}(x)\right|} \geq \alpha<1 e \frac{p_{k}(x)}{\left|\tau_{K}^{\prime}(x)\right|}$ pertença ao intervalo da função associado ao valor de $x$, então, o mapa randômico $T$ admite um número finito (pelo menos uma) de medidas invariantes absolutamente ergódigas, ou seja, pelo menos uma fdp invariante ao mapa randômico $T$ (Bahsoun et alii, 2007).

Este teorema garante a existência de pelo menos uma $f d p$ invariante ao mapa randômico $T$ e faz com que a árvore se expanda na média, uma vez que as ramificações não se concentram em um único ponto. Esta é uma característica desejável para uma árvore binomial, principalmente aquelas utilizadas no mercado financeiro.

Assim, a árvore binomial varia a partir de um estado prévio (ou inicial) $x$. A cada passo na árvore, o processo gera $K$ diferentes possíveis valores $\tau_{1}(x), \ldots, \tau_{K}(x)$ com probabilidades $p_{1}(x), \ldots, p_{K}(x)$, respectivamente para cada posição (estado) $x$. Os preços $s(n)$ do ativo analisado são normalizados para ter um limite superior $M$, com $0<M<1$, tal que $0<s(n)<M$. Estes modelos em tempo discreto podem ser utilizados para se estimar os modelos contínuos ao longo de um período de tempo finito.

Os estados e as probabilidades dependem dos estados prévios $x$ por meio das funções $\tau_{K}(x)$ e $p_{K}(x)$, portanto são dependentes da posição. Representa-se o conjunto destas transformações como $T_{n}(x)=\tau_{1}(x), \ldots, \tau_{k}(x) ; p_{1}(x), \ldots, p_{k}(x)$, onde $\tau_{u}(x)=u(x) * x$ e $\tau_{d}(x)=d(x) * x$.

Por exemplo, se o prêmio do risco (opção) normalizado no tempo $n=0$ é 0,3 ; então as possíveis trajetórias dos preços nos tempos $n=1,2$ são dadas por:

Para cada valor inicial tem-se uma árvore binomial diferente com o mesmo formato da apresentada acima, caracterizando um sistema dinâmico estocástico. Dado um determinado valor de $x$, a árvore descreve todos os possíveis passos (estados) dos preços que o ativo pode assumir de uma maneira semelhante às árvores binomiais clássicas. Estas árvores se expandem e se contraem de acordo como 
as variações dos valores iniciais, contrariamente aos modelos clássicos em que os parâmetros, como por exemplo a volatilidade, permanecem imutáveis para todos os valores iniciais.

As probabilidades dos preços podem aumentar (ou diminuir) no próximo estado, dependendo, por exemplo, de como os preços variam. Isto também se aplica às variações dos preços, ou seja, as variações dos preços são em função dos preços correntes. O conceito de mapas randômicos utilizado também permite introduzir saltos (jumps) dependentes da posição que podem ser percebidos a partir da análise das leis de up's e down's.

Agora, vamos aplicar o Teorema 3.1 para verificar se o mapa randômico acima permite uma $f d p f$ invariante a $T$.

$$
\sup _{x} \frac{p_{u}(x)}{\left|\tau_{u}^{\prime}(x)\right|}+\sup _{x} \frac{p_{d}(x)}{\left|\tau_{d}^{\prime}(x)\right|}=0,54+0,44<1
$$

O mapa randômico anterior satisfaz os pressupostos do Teorema 3.1.

\subsection{Matrizes $M_{u}, M_{d}$ e $M$ associadas aos mapas randômicos}

As matrizes $M_{u}, M_{d}$ e $M$ serão estimadas, de acordo com as definições e os teoremas apresentados em Góra e Boyarsky (2003) e Bahsoun et alii (2007), para caracterizar a forma da função de densidade de probabilidade estacionária associada ao modelo binomial ajustado via mapas randômicos. Isto se torna possível porque uma função de densidade de probabilidade invariante tem matrizes constantes em cada seção. Este é um bom recurso uma vez que permitirá aproximar facilmente qualquer $f d p$ invariante associada a um modelo binomial por meio de mapas randômicos.

A partir do exemplo anterior, caso seja definida uma partição comum $\left.[i / 10,(i+1) / 10)_{i=0}^{9}\right]$, podemos representar as variações deste processo por meio de matrizes de subida $M_{u}$ e de descida $M_{d}$ ou através da matriz $M$ que representa o mapa randômico das transformações.

Em seguida são apresentadas as matrizes $M_{u}, M_{d}$ e $M$ para o exemplo anterior.

$$
M_{u}=\left\{\begin{array}{cccccccccc}
0,71 & 0,71 & 0 & 0 & 0 & 0 & 0 & 0 & 0 & 0 \\
0 & 0 & 0,71 & 0,71 & 0 & 0 & 0 & 0 & 0 & 0 \\
0 & 0 & 0 & 0 & 0,71 & 0,71 & 0 & 0 & 0 & 0 \\
0 & 0 & 0 & 0 & 0 & 0 & 0,87 & 0,87 & 0 & 0 \\
0 & 0 & 0 & 0 & 0 & 0 & 0 & 0 & 0,87 & 0,87 \\
0 & 0 & 0 & 0 & 0 & 0 & 0 & 0,87 & 0,87 & 0 \\
0 & 0 & 0 & 0 & 0 & 0 & 0 & 0,91 & 0 & 0 \\
0 & 0 & 0 & 0 & 0 & 0 & 0 & 0,91 & 0 & 0 \\
0 & 0 & 0 & 0 & 0 & 0 & 0 & 0 & 0,91 & 0 \\
0 & 0 & 0 & 0 & 0 & 0 & 0 & 0 & 0 & 0,91
\end{array}\right\}
$$




$$
M_{d}=\left\{\begin{array}{cccccccccc}
1,11 & 0 & 0 & 0 & 0 & 0 & 0 & 0 & 0 & 0 \\
1,11 & 0 & 0 & 0 & 0 & 0 & 0 & 0 & 0 & 0 \\
0 & 1,11 & 0 & 0 & 0 & 0 & 0 & 0 & 0 & 0 \\
0 & 1,11 & 0 & 0 & 0 & 0 & 0 & 0 & 0 & 0 \\
0 & 0 & 1,11 & 0 & 0 & 0 & 0 & 0 & 0 & 0 \\
0 & 0 & 1,11 & 0 & 0 & 0 & 0 & 0 & 0 & 0 \\
0 & 0 & 0 & 1,11 & 0 & 0 & 0 & 0 & 0 & 0 \\
0 & 0 & 0 & 0 & 0 & 0 & 1,11 & 1,11 & 0 & 0 \\
0 & 0 & 0 & 0 & 0 & 0 & 0 & 1,11 & 1,11 & 0 \\
0 & 0 & 0 & 0 & 0 & 0 & 0 & 0 & 1,11 & 1,11
\end{array}\right\}
$$

Os valores de $p_{u}(x)$ e $p_{d}(x)$ são:

$$
p_{u}(x)=[0,750,750,750,650,650,650,650,600,600,60] .
$$

$\mathrm{e}$

$$
p_{d}(x)=[0,250,250,250,350,350,350,350,400,400,40] .
$$

Agora podemos encontrar a matriz $M$ associada ao mapa randômico das transformações apresentado anteriormente. Assim, partiu-se do conhecimento a priori sobre o ativo, construímos as equações do mapa randômico e agora iremos estimar a matriz $M$ que sintetiza todas as transformações, ou seja, as variações possíveis do ativo. Posteriormente vamos determinar a fdp associada ao modelo do ativo ajustado.

$$
M_{d}=\left\{\begin{array}{cccccccccc}
0,8100 & 0,5325 & 0 & 0 & 0 & 0 & 0 & 0 & 0 & 0 \\
0,2775 & 0 & 0,5325 & 0,5325 & 0 & 0 & 0 & 0 & 0 & 0 \\
0 & 0,2775 & 0 & 0 & 0,5325 & 0,5325 & 0 & 0 & 0 & 0 \\
0 & 0,3885 & 0 & 0 & 0 & 0 & 0,5655 & 0,5655 & 0 & 0 \\
0 & 0 & 0,3885 & 0 & 0 & 0 & 0 & 0 & 0,5655 & 0,5655 \\
0 & 0 & 0,3885 & 0 & 0 & 0 & 0 & 0,5655 & 0,5655 & 0 \\
0 & 0 & 0 & 0,3885 & 0 & 0 & 0 & 0,5915 & 0 & 0 \\
0 & 0 & 0 & 0 & 0 & 0 & 0,4400 & 0,9900 & 0 & 0 \\
0 & 0 & 0 & 0 & 0 & 0 & 0 & 0,4440 & 0,9840 & 0 \\
0 & 0 & 0 & 0 & 0 & 0 & 0 & 0 & 0,4440 & 0,9900
\end{array}\right\}
$$

A partir desta matriz $M$ podemos encontrar uma $f d p$ estacionária $f$, a partir da equação $f M=f$, associada ao mapa randômico apresentado anteriormente. Seja

$$
f=\left(f_{1}, f_{2}, f_{3}, f_{4}, f_{5}, f_{6}, f_{7}, f_{8}, f_{9}, f_{10}\right), f i=f \mid I_{i}, \operatorname{com} i=1,2, \ldots, 9,10
$$

fazendo

$$
f_{1}+f_{2}+f_{3}+f_{4}+f_{5}+f_{6}+f_{7} f_{8}+f_{9}+f_{10}=1
$$


Satisfazendo à equação $f M=f$. Então,

$$
\begin{aligned}
& f_{1}=0,0406, f_{2}=0,0278, f_{3}=0,0253, f_{4}=0,0025, f_{5}=0,0135 \\
& f_{6}=0,0135, f_{7}=0,0445, f_{8}=0,0688, f_{9}=0,0016, f_{10}=0,7619
\end{aligned}
$$

Observa-se que a $f d p$ estimada $f$ é constante para cada intervalo, logo quanto mais fina é a partição melhor será a aproximação.

\subsection{O problema inverso de Perron-Frobenius e a árvore binomial implícita}

Neste ponto do artigo surge a seguinte questão: será que se pode construir uma matriz $M$ da qual podemos extrair um mapa randômico $T$ e chamá-lo de uma árvore binomial implícita com função de densidade de probabilidade estacionária $f$ ? Sim, caso $f M=f$, onde $M$ é a matriz das transformações associada ao mapa randômico $T$, então este mapa define uma árvore binomial implícita com $f d p f$. Trata-se do problema inverso de Perron-Frobenius, mas ainda falta definir o conceito de transformação $\mathrm{N}$-banda. $\mathrm{O}$ teorema que se segue garante a existência desta árvore binomial implícita.

Theorem 3.2 Seja $T$ uma transformação na partição uniforme $I \rightarrow I$ de $N$ elementos em $R$, com $N=2 s+1$ e matriz Perron-Frobenius dada por $M_{\text {tau }}=$ $\left(\pi_{i j}\right)$. Dado um vetor probabilístico $f=\left(f_{1}, \ldots, f_{N}\right)$ qualquer com $f_{i}>0, i=$ $1, \ldots, N$ que corresponde a uma função de densidade de probabilidade. Caso

$$
f_{i} \pi_{i, j}=f_{j} \pi_{j, i}
$$

Para qualquer $1 \geq i, j \geq N$, então as densidades correspondentes ao vetor $f$ são invariantes a $T$. O teorema a seguir liga a $f d p f$ ao modelo binomial construído a partir de um mapa randômico $T$.

Theorem 3.3 Seja $f=\left(f_{1}, \ldots, f_{N}\right)$ com valores constantes em cada partição nos reais de $I=[a, b]$ dividido em $N$ intervalos iguais. Então existe um mapa randômico $T$ tal que

$$
\sup _{x} \frac{p_{u}(x)}{\left|\tau_{u}^{\prime}(x)\right|}+\sup _{x} \frac{p_{d}(x)}{\left|\tau_{d}^{\prime}(x)\right|} \geq \alpha<1
$$

$\operatorname{com} \tau_{u}(x) \leq x, \tau_{d}(x) \geq x$ e $f$ sendo invariante a $T$. 
A prova destes teoremas são apresentadas em Bahsoun et alii (2007) e eles fornecem um caminho para construir uma matriz probabilística $N$-banda que preserva $f$, mas não garantem a unicidade de $M$ que ainda é um problema em aberto.

Vamos agora apresentar um exemplo para ilustrar a construção das matrizes $M, M_{u}, M_{(d)}$ e dos vetores $p_{u}, p_{d}$. Ou seja, como extrair um mapa randômico $T$, a partir de uma função de densidade de probabilidade estacionária $f$. A $f d p$ é a gaussiana dada por $f(x)=x * \exp (-x / 2), \operatorname{com} x=i, \ldots, i+1$. Pode-se escolher livremente as constantes $s \geq N-1, c_{1}, c_{2}, \ldots, c_{s}$ tal que $c_{1}+c_{2}+\ldots+c_{s} \geq 1 \mathrm{e}$ $d_{1}, d_{2}, \ldots, d_{s}$ tal que $0<d_{i}<1,1 \geq i \geq s$.

Estas constantes têm um efeito direto sobre a árvore binomial ajustada. A constante $s$ é o número de intervalos adjacentes que podem ser alcançados em um passo a partir de qualquer posição em qualquer direção. Quanto maior for o valor de $s$, maior é o salto que pode ser obtido em um passo. As constantes $c$ 's e $d$ 's estão relacionadas com as probabilidades do salto acontecer a partir de uma determinada posição. Assim, pequenos valores de c's e d's implicam menores probabilidades de se ter o salto. Logo, pequenos valores de s, $c$ 's e $d$ 's, ao mesmo tempo, torna as trajetórias menos variáveis. Já grandes valores de $s, c$ 's e d's geram trajetórias com maiores flutuações.

Em relação aos valores dos c's e d's, fazemos os seguintes comentários adicionais:

1. Estes c's e d's são utilizados para produzir os valores da matriz M tal que $\mathrm{fM}=\mathrm{f}$.

2. Os valores $c$ 's e d's determinam as probabilidades de subida (up) e de descida (down) do mapa randômico $T$. Podemos verificar que se $c$ 's e $d$ 's são pequenos o mapa randômico não vai causar mudanças muito grande. $\mathrm{O}$ oposto é verdadeiro.

Em seguida apresenta-se um exemplo de matriz $N$-banda especificada a partir da $f d p$ gaussiana definida anteriormente. Esta matriz é estimada para uma partição comum $\left.[i / 10,(i+1) / 10)_{i=0}^{9}\right]$ :

Sejam $N=10 ; s=3 ; c_{1}=0,6 ; c_{2}=0,2 ; c_{3}=0,1 ; d_{1}=0,9 ; d_{2}=0,85 ;$ $d_{3}=0,95$.

Aplicando um algoritmo para a construção das matrizes, temos:

1. A escolha de $p_{u}(x)$ e $p_{d}(x)$ pode ser feita dentro de um intervalo da forma $\left[L\left(c_{i}, f_{i}\right), H\left(c_{i}, f_{i}\right)\right]$, desde que obedeçamos às condições impostas anteriormente. Nesta fase da especificação de $M$ pode também ser incorporado o conhecimento a priori sobre o ativo.

2. Finalmente, a matriz $M$ de Perron-Frobenius que define o mapa randômico $T$ pode ser construída a partir de $f$. 
A matriz $M$ de Perron-Frobenius é definida em seguida:

$$
M_{d}=\left\{\begin{array}{cccccccccc}
0,10 & 0,60 & 0,20 & 0,10 & 0 & 0 & 0 & 0 & 0 & 0 \\
0,59 & 0,04 & 0,25 & 0,08 & 0,04 & 0 & 0 & 0 & 0 & 0 \\
0,23 & 0,29 & 0,05 & 0,29 & 0,10 & 0,05 & 0 & 0 & 0 & 0 \\
0,15 & 0,12 & 0,37 & 0,04 & 0,22 & 0,07 & 0,04 & 0 & 0 & 0 \\
0 & 0,08 & 0,17 & 0,29 & 0,05 & 0,28 & 0,09 & 0,05 & 0 & 0 \\
0 & 0 & 0,12 & 0,14 & 0,38 & 0,04 & 0,22 & 0,07 & 0,04 & 0 \\
0 & 0 & 0 & 0,10 & 0,18 & 0,31 & 0,04 & 0,24 & 0,08 & 0,04 \\
0 & 0 & 0 & 0 & 0,13 & 0,15 & 0,36 & 0,07 & 0,21 & 0,07 \\
0 & 0 & 0 & 0 & 0 & 0,11 & 0,17 & 0,32 & 0,16 & 0,24 \\
0 & 0 & 0 & 0 & 0 & 0 & 0,13 & 0,16 & 0,35 & 0,36
\end{array}\right\}
$$

Como se trata do problema inverso de Perron-Frobenius tem-se que decompor $M$ em $M_{u}, M_{d}, p_{u}$ e $p_{d}$ e surge um problema de fatoração $M_{u}, M_{d}, p_{u}$ e $p_{d}$ de $M$ tal que:

$$
M=p_{u} M_{u}+p_{d} M_{d}
$$

Os valores das matrizes $M_{u}$ e $M_{d}$ são apresentados logo em seguida.

$$
M_{u}=\left\{\begin{array}{cccccccccc}
0,07 & 0,61 & 0,21 & 0,10 & 0 & 0 & 0 & 0 & 0 & 0 \\
0 & 0,07 & 0,61 & 0,21 & 0,10 & 0 & 0 & 0 & 0 & 0 \\
0 & 0 & 0,07 & 0,61 & 0,21 & 0,10 & 0 & 0 & 0 & 0 \\
0 & 0 & 0 & 0,07 & 0,61 & 0,21 & 0,10 & 0 & 0 & 0 \\
0 & 0 & 0 & 0 & 0,07 & 0,61 & 0,21 & 0,10 & 0 & 0 \\
0 & 0 & 0 & 0 & 0 & 0,07 & 0,61 & 0,21 & 0,10 & 0 \\
0 & 0 & 0 & 0 & 0 & 0 & 0,07 & 0,61 & 0,21 & 0,10 \\
0 & 0 & 0 & 0 & 0 & 0 & 0 & 0,15 & 0,64 & 0,21 \\
0 & 0 & 0 & 0 & 0 & 0 & 0 & 0 & 0,32 & 0,28 \\
0 & 0 & 0 & 0 & 0 & 0 & 0 & 0 & 0 & 1
\end{array}\right\}
$$

$$
M_{d}=\left\{\begin{array}{cccccccccc}
1 & 0 & 0 & 0 & 0 & 0 & 0 & 0 & 0 & 0 \\
0,98 & 0,02 & 0 & 0 & 0 & 0 & 0 & 0 & 0 & 0 \\
0,43 & 0,54 & 0,03 & 0 & 0 & 0 & 0 & 0 & 0 & 0 \\
0,23 & 0,19 & 0,57 & 0,02 & 0 & 0 & 0 & 0 & 0 & 0 \\
0 & 0,15 & 0,30 & 0,53 & 0,02 & 0 & 0 & 0 & 0 & 0 \\
0 & 0 & 0,18 & 0,21 & 0,59 & 0,02 & 0 & 0 & 0 & 0 \\
0 & 0 & 0 & 0,16 & 0,30 & 0,52 & 0,02 & 0 & 0 & 0 \\
0 & 0 & 0 & 0 & 0,20 & 0,23 & 0,54 & 0,03 & 0 & 0 \\
0 & 0 & 0 & 0 & 0 & 0,17 & 0,27 & 0,49 & 0,07 & 0 \\
0 & 0 & 0 & 0 & 0 & 0 & 0,17 & 0,21 & 0,47 & 0,14
\end{array}\right\}
$$


Os valores de $p_{u}(x)$ e $p_{d}(x)$ são:

$$
p_{u}(x)=[0,970,400,470,350,450,350,390,340,350,25]
$$

e

$$
p_{d}(x)=[0,030,600,530,650,550,650,610,660,650,75]
$$

Para verificar se as matrizes foram calculadas corretamente, utiliza-se a seguinte equação:

$$
\text { Erro }=M-\left(p_{u} M_{u}+p_{d} M_{d}\right)
$$

A matriz Erro resultou na matriz nula, garantindo que as matrizes foram calculadas corretamente. É importante observar que as matrizes acima tiveram seus valores arredondados, mas nos cálculos computacionais a precisão é de quinze casas decimais.

Este método de transformações aplica-se aos mapas aleatórios com a mesma facilidade com que se aplica aos mapas deterministas comuns. Dada uma densidade invariante $f$ para um mapa aleatório, podemos construir uma matriz de transformação $M$ e esta transformação têm a sua função densidade estacionária $f$ (Góra e Boyarsky, 1993, 2003).

A matriz de transformação $M$ introduzida é importante porque proporciona um relacionamento claro e único entre a função de densidade invariante $f$ e a própria transformação $M$. Entretanto, no caso do problema inverso, a transformação $M$ não é única para uma dada função de densidade $f$ e ainda constitui um problema em aberto. A Figura 1 apresenta a lei de up resultante do mapa randômico extraído sobre o intervalo $[0,1]$. 


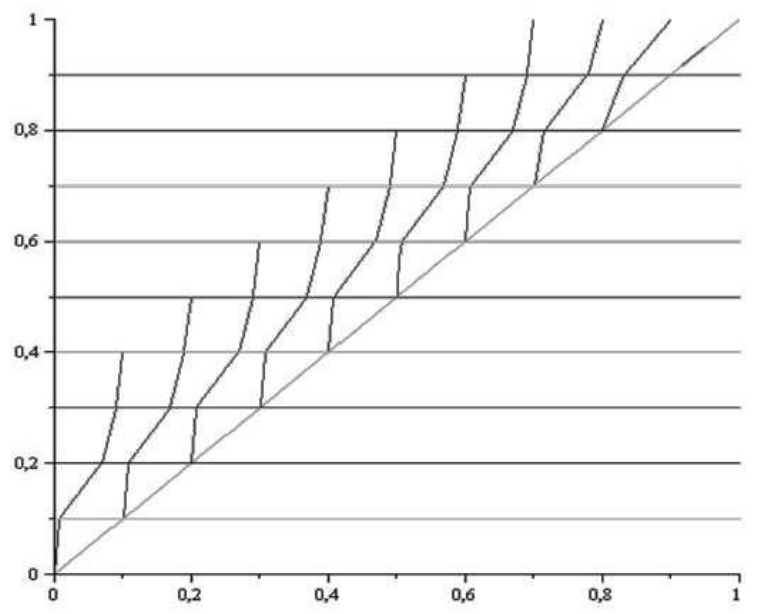

Figura 1

Lei de up sobre o intervalo $[0,1]$

Observa-se na figura anterior que os valores dos saltos a partir do intervalo $[0,70,8]$ até $[0,91]$ são menores que os anteriores, logo nesta região as subidas são um pouco atenuadas.

A Figura 2 apresenta os resultados obtidos a partir da lei de down do mapa randômico extraído sobre o intervalo $[0,1]$.

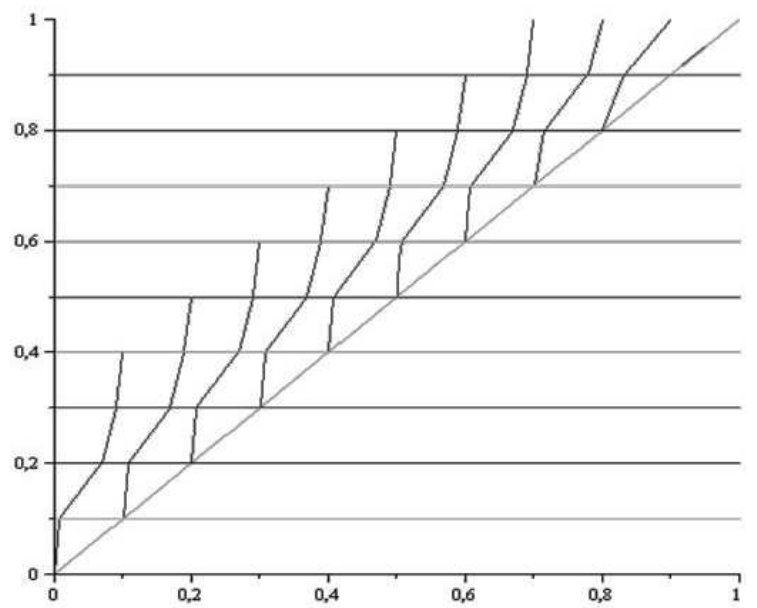

Figura 2

Lei de down sobre o intervalo $[0,1]$ 
Observa-se na figura anterior que os valores dos saltos a partir do intervalo $[00,1]$ até $[0,20,3]$ são menores que os valores dos outros intervalos, logo nesta região as quedas são atenuadas. Na aplicação anterior, geralmente as leis de up's e down's apresentam saltos (jumps) para cima e para baixo com amplitude significativa.

\section{Aplicação da Metodologia no Mercado Brasileiro de Opções}

O ativo analisado nesta seção é a opção de compra VALEI sobre a ação da Vale tipo VALEPNAN1. Os dados analisados são das cotações do dia 21/09/2009 com vencimento em 19/10/2009. O gráfico das freqüências normalizadas versus o preço de exercício (Strike) é apresentado na Figura 3, abaixo.

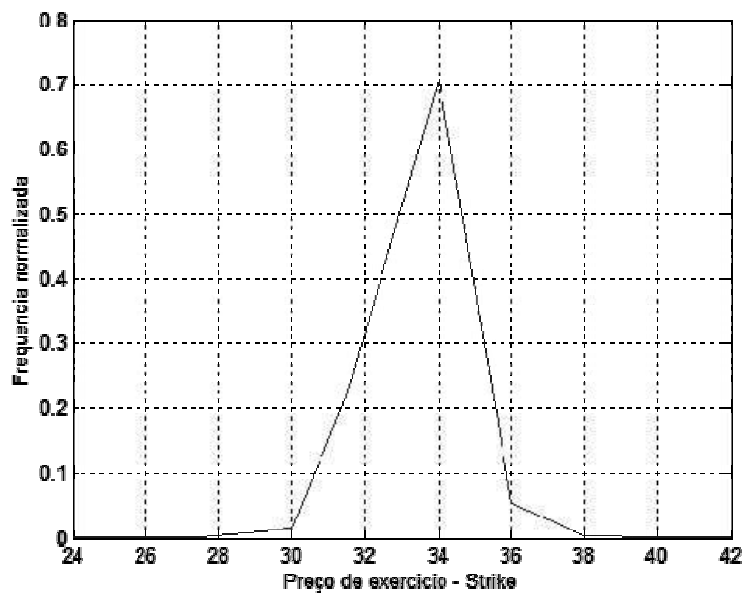

Figura 3

$F d p$ não paramétrica do preço de exercício da opção VALEI

A matriz $N$-banda estimada a partir da fdp extraída do ativo acima ilustra empiricamente a capacidade do método de resolver o problema inverso de PerronFrobenius para uma $f d p$ não paramétrica. Seja os valores da $F d p$ do preço de exercício da opção VALEI:

$$
\begin{aligned}
f_{1} & =3.00089 E-07 ; f_{2}=5.03154 E-05 ; f_{3}=9.73767 E-05 \\
f_{4} & =0.015208217 ; f_{5}=0.218798676 ; f_{6}=0.708061811 ; \\
f_{7} & =0.055301499 ; f_{8}=0.002475997 ; f_{9}=5.76575 E-06 ; \\
f_{10} & =4.21473 E-08
\end{aligned}
$$

Para os respectivos preços de exercícios desta opção: 


$$
\begin{aligned}
& p_{1}=24 ; p_{2}=26 ; p_{3}=27,48 ; p_{4}=30 ; f_{5}=31,48 \\
& f_{6}=34 ; f_{7}=36 ; f_{8}=38 ; f_{9}=40 ; f_{10}=42
\end{aligned}
$$

A matriz $N$-banda é definida para uma partição comum $\left.[i / 10,(i+1) / 10)_{i=0}^{9}\right]$ $\operatorname{com} N=10 ; s=2 ; c_{1}=0,3, c_{2}=0,1, d_{1}=0,5 ; d_{2}=0,4$. Destaca-se que os valores dos $c$ 's, $d$ 's e s não são muito grandes, logo os saltos e as flutuações são menores.

A matriz $M$ de Perron-Frobenius é definida em seguida:

$$
M=\left\{\begin{array}{cccccccccc}
0,60 & 0,30 & 0,10 & 0 & 0 & 0 & 0 & 0 & 0 & 0 \\
0 & 0,60 & 0,30 & 0,10 & 0 & 0 & 0 & 0 & 0 & 0 \\
0 & 0,15 & 0,51 & 0,25 & 0,08 & 0 & 0 & 0 & 0 & 0 \\
0 & 0 & 0,002 & 0,60 & 0,30 & 0,10 & 0 & 0 & 0 & 0 \\
0 & 0 & 0 & 0,02 & 0,60 & 0,30 & 0,10 & 0 & 0 & 0 \\
0 & 0 & 0 & 0 & 0,01 & 0,88 & 0,02 & 0 & 0 & 0 \\
0 & 0 & 0 & 0 & 0 & 0,40 & 0,40 & 0,30 & 0,01 & 0 \\
0 & 0 & 0 & 0 & 0 & 0,40 & 0,30 & 0,30 & 0 & 0 \\
0 & 0 & 0 & 0 & 0 & 0 & 0,40 & 0,30 & 0,30 & 0 \\
0 & 0 & 0 & 0 & 0 & 0 & 0 & 0,40 & 0,30 & 0,30
\end{array}\right\}
$$

Resolvendo o problema inverso de Perron-Frobenius tem-se que decompor $M$ em $M_{u}, M_{d}, p_{u}$ e $p_{d}$ :

$$
M=p_{u} M_{u}+p_{d} M_{d}
$$

Os valores das matrizes $M_{u}$ e $M_{d}$ são apresentados logo em seguida.

Os valores de $p_{u}(x)$ e $p_{d}(x)$ são:

$$
p_{u}(x)=[0,820,820,690,820,800,640,220,210,210,21] .
$$

$\mathrm{e}$

$$
p_{d}(x)=[0,180,180,310,180,200,360,780,790,790,79] .
$$

Verificando se as matrizes foram calculadas corretamente:

$$
\text { Erro }=M-\left(p_{u} M_{u}+p_{d} M_{d}\right)
$$

A matriz Erro resultou na matriz nula, garantindo que as matrizes foram calculadas corretamente. É importante observar que as matrizes acima tiveram seus valores arredondados, mas nos cálculos computacionais a precisão é de quinze casas decimais. $[0,1]$.

A Figura 4 apresenta a função $u p$ que resulta desta aplicação sobre o intervalo 


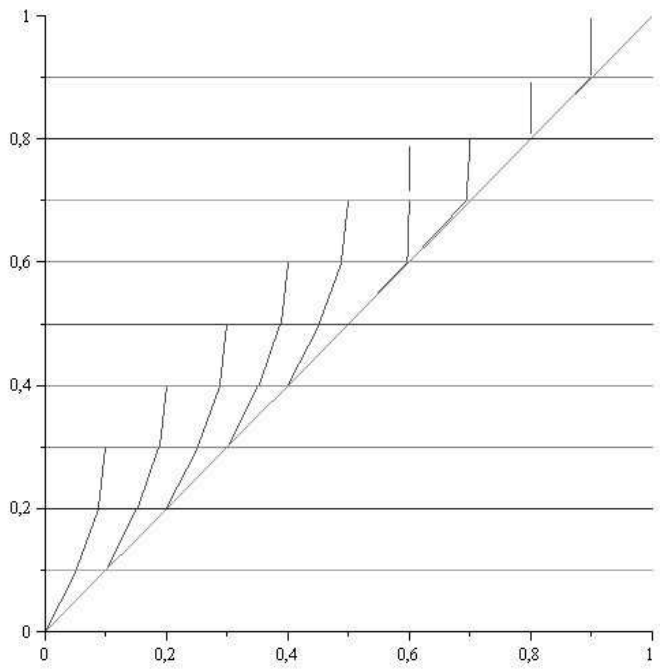

Figura 4

Função de $u p$ sobre o intervalo $[0,1]$

Fdp não paramétrica do preço de exercício da opção VALEI

Já a Figura 5 abaixo ilustra a função down sobre o intervalo $[0,1]$.

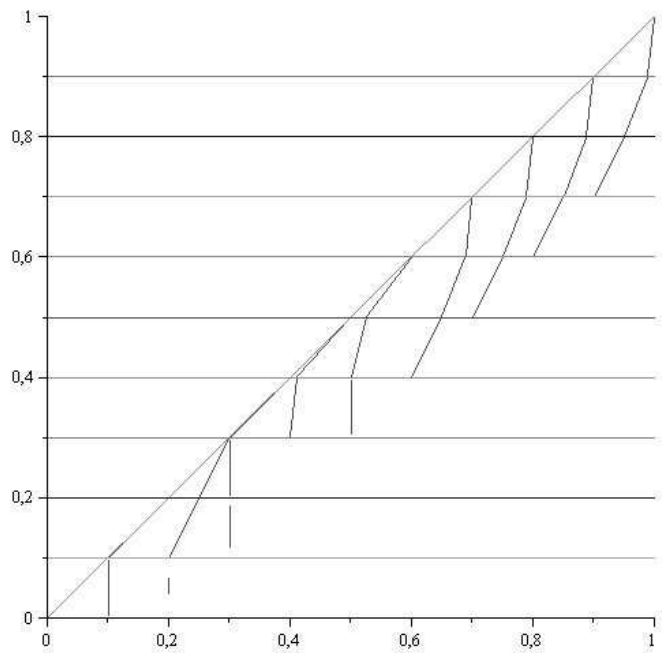

Figura 5

Função de down sobre o intervalo $[0,1]$ 
Os saltos são pequenos, o que já era esperado, já que os valores dos $s, c$ 's e d's são menores.

\section{Comentários}

Foi apresentado que é possível a construção de um mapa randômico dependente da posição a partir do conhecimento a priori sobre uma variável aleatória associada a um investimento analisado. A partir deste mapa randômico podese estimar uma função de densidade de probabilidade invariante e única. As transformações introduzidas são importantes porque proporcionam um relacionamento entre a função de densidade invariante $f$ e as transformações $M$. Este método aplica-se aos mapas aleatórios e deterministas comuns.

A aplicação que utiliza os preços da opção VALEI ilustra a construção de uma árvore binomial implícita a partir de uma função de densidade de probabilidade discreta estacionária. Esta árvore binomial construída tem características de árvore binomial generalizada (Jackwerth, 1997). Entretanto, neste caso, a matriz $M$ das transformações não é única e a questão da sua singularidade ainda é um problema em aberto.

\section{Referências}

Arrow, K. J. \& Hahn, F. H. (1971). General Competitive Analysis. Holden-Day, San Francisco.

Bahsoun, W., Góra, P., Mayoral, S., \& Morales, M. (2007). Random dynamics and finance: Constructing implied binomial trees from a predetermined stationary density. Applied Stochastic Models in Business and Industry, 23:181-212.

Barle, S. \& Cakici, N. (1998). How to grow a smiling tree. Journal of Financial Engineering, 7:127-146.

Black, F. \& Scholes, M. (1973). Pricing of options and corporate liabilities. Journal of Political Economy, 81:637-659.

Chang, E. J. \& Tabak, B. M. (2002). Risk neutral probability densities. Financial Stability Report, Department of Research, Banco Central do Brasil.

Cox, J. C., Ross, S. A., \& Rubinstein, M. (1979). Option pricing: A simplified approach. Journal of Financial Economics, 7:229-264.

Debreu, G. (1959). Theory of Value. Wiley, New York.

Derman, E. \& Kani, I. (1994). The volatility smile and its implied tree. Quantitative Strategies Research Notes, Goldman Sachs.

Dupire, B. (1994). Pricing with a smile. Risk, 7:18-20. 
Frisch, R. (1933). Propagation problems and impulse problems in dynamic economics. In Gordon, R. A. \& Klein, L. R., editors, Economic Essays in Honor of Gustav Cassel, pages 171-205. Allen \& Unwin, London.

Frisch, R. (1936). On the notion of equilibrium and disequilibrium. Review of Economic Studies, 3:100-105.

Góra, P. \& Boyarsky, A. (1993). A matrix solution to the inverse Perron-Frobenius problem. Proceedings of the American Mathematical Society, 118:409-414.

Góra, P. \& Boyarsky, A. (2003). Absolutely continuous invariant measures for random maps with position dependent probabilities. Journal of Mathematical Analysis and Applications, 278:225-242.

Grandmont, J. M. (1977). Temporary general equilibrium theory. Econometrica, 45:535-572.

Grandmont, J. M. (1982). Temporary general equilibrium theory. In Arrow, K. J. \& Intriligator, M. D., editors, Handbook of Mathematical Economics, chapter 19. Elsevier, Amsterdam, North-Holland.

Grandmont, J. M. \& Hildenbrand, W. (1974). Stochastic processes of temporary equilibria. Journal of Mathematical Economics, 1:247-277.

Jackwerth, J. G. (1997). Generalized binomial trees. The Journal of Derivatives, $5: 7-17$.

Merton, R. C. (1973). Theory of rational option pricing. Bell Journal of Economics, 4:141-183.

Rubinstein, M. (1994). Implied binomial trees. Journal of Finance, 49:771-818.

Schenk-Hoppé, K. R. (2000). Random dynamical systems in economics. Institute for Empirical Research in Economics, University of Zurich, ISSN 1424-0459, Working Paper no. 67. 